\title{
Evanescence Meets Elegance: Story of Invisible Sweet Marker
}

\author{
Satish Ramanathan ${ }^{1}$, Srinivas N Chakravarthy ${ }^{2}$, Smitha $\mathrm{S}^{3}$, Kavitha $\mathrm{S}^{4}$
}

\begin{abstract}
Aim: To explore the interference of a hemoglobin $\mathrm{E}$ on glycated hemoglobin ( $\mathrm{HbA1c}$ ) measurement on two methodologies including high performance liquid chromatography (HPLC) and immunoassay.

Background: $\mathrm{HbA} 1 \mathrm{c}$ is an invincible analyte in modern laboratory medicine era. The role of $\mathrm{HbA} 1 \mathrm{c}$ in diabetes mellitus has expanded from prognosis to diagnosis of diabetes. Hence, it becomes an essential responsibility of the medical testing laboratories to deliver quality results to the patients. The quality of a result is determined by several factors. One essential determinant of the quality of an analyte in a clinical laboratory is the choice of method of testing. $\mathrm{HbA} 1 \mathrm{c}$ has various standardized methods of measurement, each of it having its own advantages and limitations. One significant limitation is interference from abnormal hemoglobins.
\end{abstract}

Case description: In our case report, we have tried to explore the interference of hemoglobin $\mathrm{E}$ on $\mathrm{HbA} 1 \mathrm{c}$ measurement on two methodologies including HPLC and immunoassay. In our case scenario, HbA1c immunoassay could produce a reliable HbA1c result, while HPLC was significantly being interfered by the hemoglobin variant.

Conclusion: With respect to $\mathrm{HbA} 1 \mathrm{c}$, each method has its own advantages and limitations. It is the responsibility of the laboratories to understand, adopt, and optimally utilize these methods based on the needs.

Clinical significance: $\mathrm{HbA} 1 \mathrm{c}$ is the gold standard investigation for monitoring patients with diabetes mellitus. National Glycohemoglobin Standardization Program (NGSP) provides traceability and improvement in standardization of methods used for A1C measurement. Each method has its own limitations; the most significant from the clinical significance point of view is being interfered by the abnormal hemoglobins. Hence, each laboratory must understand the strengths and limitations of the available methods in their premises and hold the responsibility for reporting clinically relevant results to its patients.

Keywords: Capillary electrophoresis, Diabetes mellitus, HbA1c, Hemoglobin E, HPLC, Immunoturbidimetry. Indian Journal of Medical Biochemistry (2020): 10.5005/jp-journals-10054-0132

\section{BACKGROUND}

A 58-year-old woman from West Bengal, India, not a known case of systemic hypertension and diabetes mellitus, presented with giddiness and generalized weakness for 15 days to General Medicine Department in our Hospital. The clinician provisionally diagnosed the patient with diabetes mellitus and referred her to the clinical laboratory for workup for diabetes mellitus including fasting and postprandial blood glucose (FBS and PPBS), HbA1C, and urine examination. Blood glucose was measured by glucose oxidaseperoxidase (GOD-POD) methodology in VITROS 5600 while glycated hemoglobin (HbA1c) was measured using high-performance liquid chromatography (Bio-Rad D10 HbA1c mode). Urine examination was done by integrated automated urine analyzer (Sysmex UX 2000). The laboratory findings were correlating with the clinical diagnosis of diabetes mellitus including FBS (155 mg/dL) and PPBS (229 mg/ $\mathrm{dL}$ ) and the urine examination showing the presence of glucose (3+), except for HbA1c which was "NOT REPORTABLE." A review of patient's high performance liquid chromatography (HPLC) revealed the absence of HbA1c peak, but for a "variant window" with a concentration of $92.1 \%$, appearing after $\mathrm{HbA0}$ (adult hemoglobin) window at 1.59 minutes (Fig. 1). Our laboratory shouldered a dual responsibility with respect to cracking the diagnostic code in this case. These include designing an approach to arrive at a clinically correlating $\mathrm{HbA1c}$ result and diagnosing the hemoglobin variant. This case report discusses the laboratory's methodological approach toward solving the diagnostic puzzle.

\section{Case Description}

HPLC (Bio-Rad D10, HbA1c mode) offers a reliable means of HbA1c measurement, being certified by NGSP and traceable to IFCC's \begin{tabular}{l}
\hline \hline 1,4 Department of Clinical Biochemistry, MIOT Hospitals, Chennai, Tamil \\
Nadu, India \\
${ }^{2,3}$ Department of Laboratory Medicine, MIOT Hospitals, Chennai, Tamil \\
Nadu, India \\
Corresponding Author: Satish Ramanathan, Department of Clinical
\end{tabular} Corresponding Author: Satish Ramanathan, Department of Clinical
Biochemistry, MIOT Hospitals, Chennai, Tamil Nadu, India, Phone: +91 9710467791, e-mail: satishraksha1980@gmail.com

How to cite this article: Ramanathan S, Chakravarthy SN, Smitha S, et al. Evanescence Meets Elegance: Story of Invisible Sweet Marker. Indian J Med Biochem 2020;24(1):37-41.

Source of support: Nil

Conflict of interest: None

reference method. ${ }^{1}$ HPLC is a charge-based method of separation of hemoglobin fractions including $\mathrm{HbA1c}$ which is expressed as a percentage (\%) of total hemoglobin present in the patient's EDTA whole blood. Though reliable in terms of precision and accuracy, it is prone to interferences from "hemoglobin variants." In our case scenario, we came across one such variant which had resulted in false lowering of $\mathrm{HbA} 1 \mathrm{c}$ to the extent of $\mathrm{HbA} 1 \mathrm{c}$ vanishing from the chromatogram. This was evident in the form of a "variant window" seen in the HPLC chromatogram (92.1\%) at 1.59 minutes in HbA1c mode (Fig. 1). Our laboratory faced diagnostic dilemma in two aspects:

- Task 1: to find an alternate method to measure HbA1c

- Task 2: to classify the hemoglobin variant.

\section{Task 1}

The available alternate method in our laboratory for $\mathrm{HbA} 1 \mathrm{c}$ measurement was an immunoturbidimetric inhibition assay (VITROS 5600). The patient's sample was processed in VITROS 5600

(0) The Author(s). 2020 Open Access This article is distributed under the terms of the Creative Commons Attribution 4.0 International License (https://creativecommons. org/licenses/by-nc/4.0/), which permits unrestricted use, distribution, and non-commercial reproduction in any medium, provided you give appropriate credit to the original author(s) and the source, provide a link to the Creative Commons license, and indicate if changes were made. The Creative Commons Public Domain Dedication waiver (http://creativecommons.org/publicdomain/zero/1.0/) applies to the data made available in this article, unless otherwise stated. 


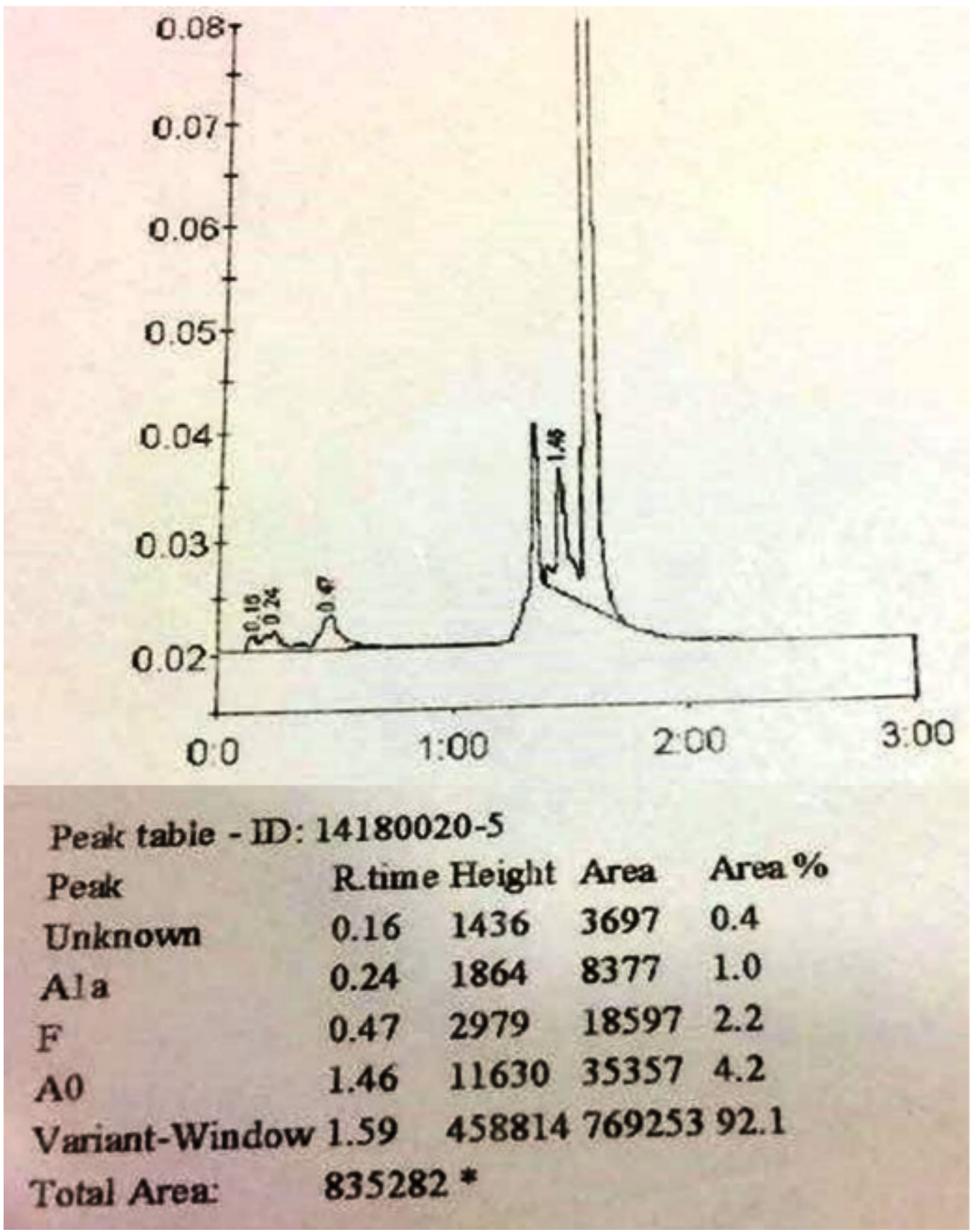

Fig. 1: HPLC HbA1c mode chromatogram

which yielded a result (7.5\%). For HbA1c of $7.5 \%$, the estimated average glucose was calculated and found to be $169 \mathrm{mg} / \mathrm{dL},{ }^{1}$ which was correlating with other laboratory findings and clinical diagnosis. Hence, $\mathrm{HbA1c}$ of $7.5 \%$ was reported for this patient carrying a remark "Abnormal hemoglobin is detected and hemoglobinopathy screening is suggested."

To understand how HbA1c on immunoassay was not interfered due to the presence of hemoglobin variant could be explained only after the hemoglobin variant was classified, for which we have proceeded to a reflective testing ${ }^{3}$ of hemoglobinopathy screening after getting consent from the patient and her treating clinician.

\section{Task 2}

Classification of hemoglobin variant, which according to the British Society of Hematology, ${ }^{4}$ required testing in two methods. Our laboratory practice included testing the patient's sample in two platforms including HPLC (Bio-Rad D10 HbA2 mode) and hemoglobin electrophoresis (Sebia Mini cap flex piercing-capillary electrophoresis). HPLC (Bio-Rad D10 HbA2 mode) showed a grossly elevated hemoglobin A2-106.8\% (biological reference interval of $\mathrm{HbA} 2-2.5-3.5 \%)^{5}$ at 3.01 minutes (Fig. 2). Studies done worldwide have shown the presence of a few common variants appearing in $\mathrm{HbA} 2$ window in $\mathrm{HPLC}$ including $\mathrm{HbE}, \mathrm{Hb}$ Lepore, $\mathrm{HbD}$ iran, etc. ${ }^{6}$ For differentiation of these variants, we proceeded 


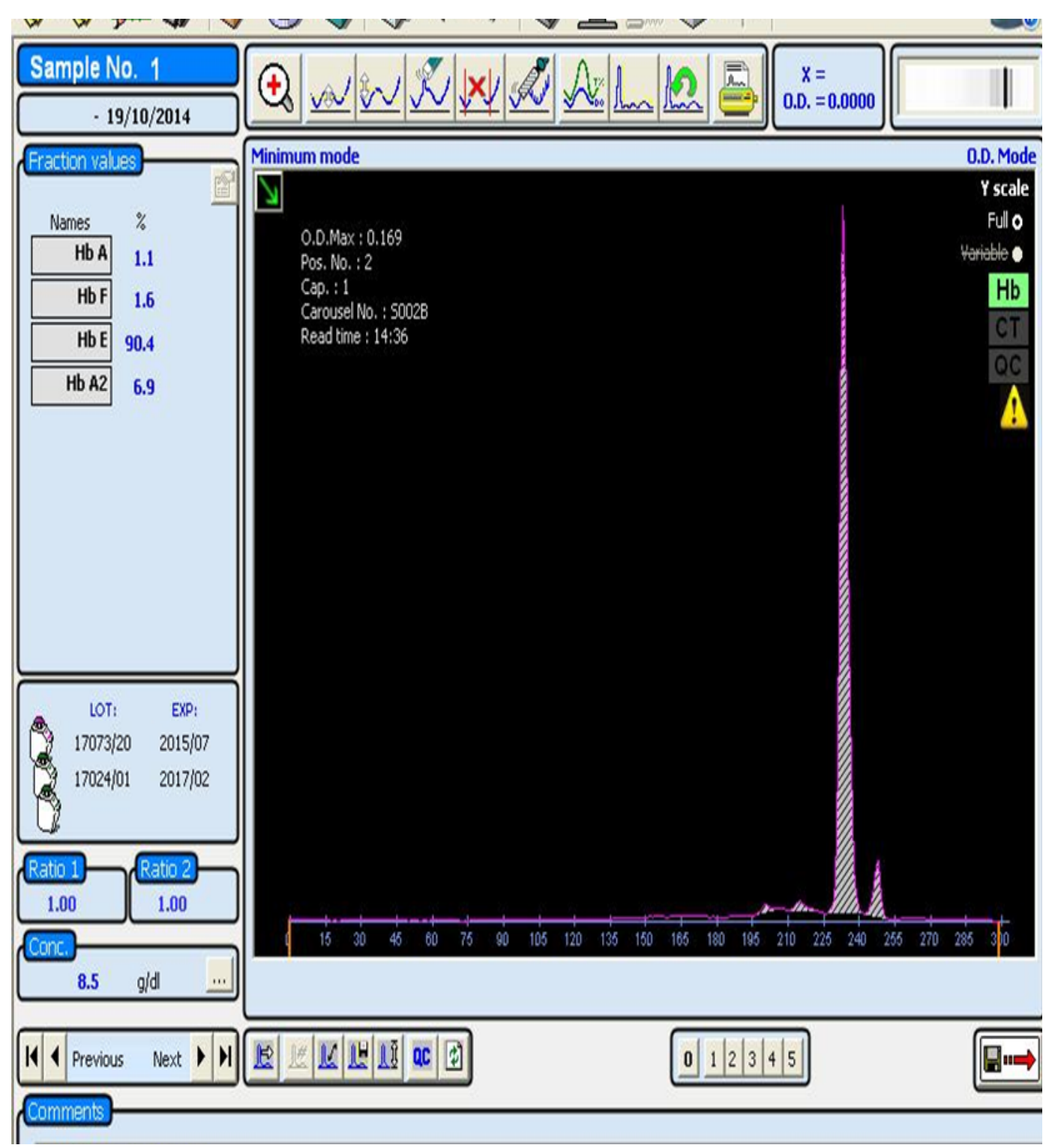

Fig. 2: HPLC HbA2F mode chromatogram

to hemoglobin electrophoresis. Capillary electrophoretogram showed a significant peak in $\mathrm{HbE}$ zone (90.4\%) and a high $\mathrm{HbA}_{2}$ (6.9\%) (Fig. 3). We did a complete blood count which showed a significant low hemoglobin-7.1 g/dL (biological reference interval: $12-15.2 \mathrm{~g} / \mathrm{dL}$ ) with microcytosis-MCV of $59.6 \mathrm{fL}$ (biological reference interval: $85-98 \mathrm{fL}){ }^{7}$ This was followed with a blood smear study which showed numerous target cells and schistiocytes. Based on these findings, we have arrived at a laboratory diagnosis of $\mathrm{HbE}$ homozygous with $\beta$-thalassemia, ${ }^{8}$ though confirmation demanded molecular studies which could not be proceeded due to unavailability of resources.

\section{Discussion}

Hemoglobin E is a clinically significant variant which is caused by a genetic single-point mutation occurring at the 26th position in the beta chain of hemoglobin where glutamate is replaced by lysine. ${ }^{8}$
VITROS immunoturbidimetric assay is based on antigen-antibody reaction, wherein a hexapeptide anti-HbA1c antibody present in the reagent binds with $\mathrm{N}$-terminal amino acids (1-6) of beta chain of hemoglobin $\mathrm{A} 0$ resulting in the formation of soluble complexes. The unbound excess anti-HbA1c antibody in the reagent is subjected to binding with polyhapten, which results in the formation of turbid complexes measured by turbidimetric assessment. The turbidity is inversely proportional to the concentration of $\mathrm{HbA1c}$ in the patient's sample. ${ }^{9}$

Since $\mathrm{HbE}$ mutation occurs away from the N-terminal of beta chain, amino acids positioned 1-6 are not affected by this mutation. This provides a scientific explanation behind the lack of interference by this variant on HbA1c immunoassay (VITROS), ${ }^{10}$ though causal relationship could be established through molecular studies, respectively. Hence, VITROS immunoassay provides a reliable assessment of A1c even during the presence of $\mathrm{HbE}$ variant. 


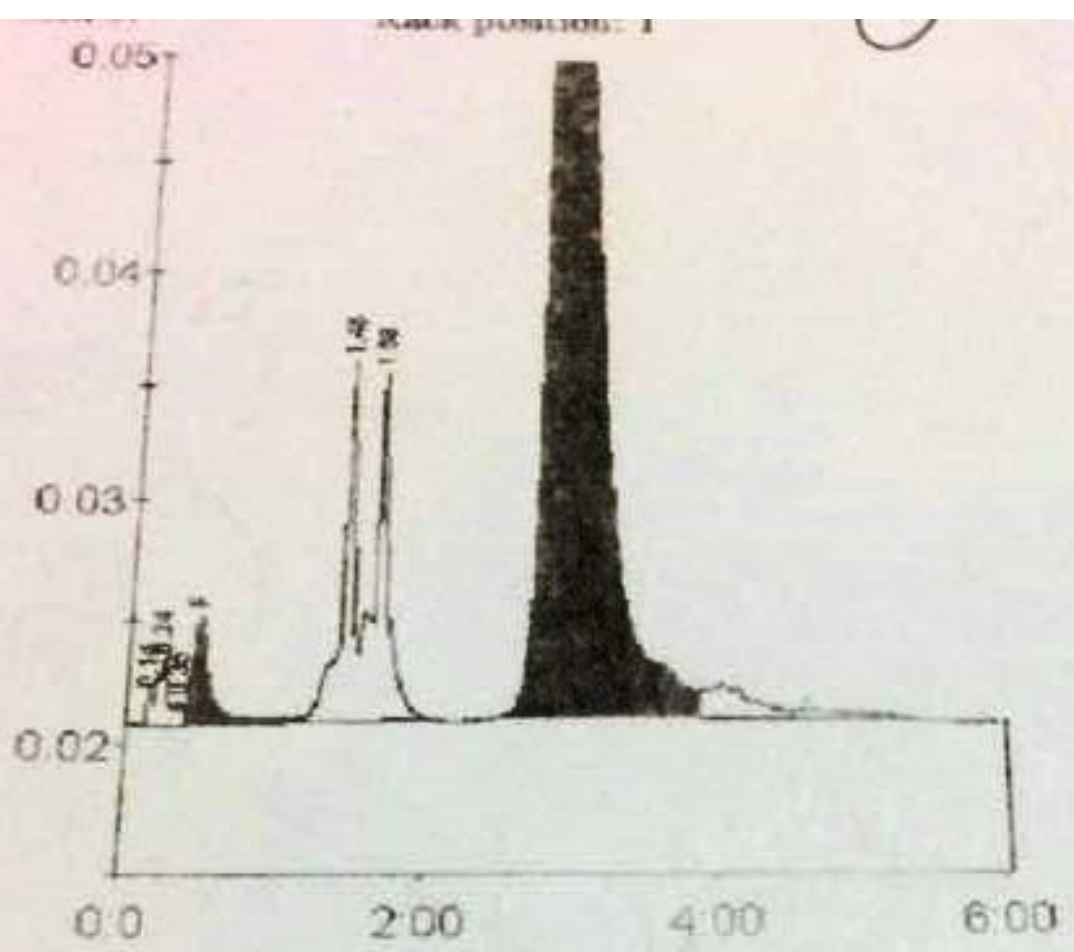

\section{Penk table - ID: $14180020-5$}

\begin{tabular}{llllll} 
Pexk & \multicolumn{3}{l}{ R.time Height Area } & Area \% \\
Unknown & 0.14 & 1502 & 3682 & 0.3 \\
Ala & 0.24 & 2727 & 12122 & 0.9 \\
Alb & 0.35 & 929 & 3689 & 0.3 \\
F & 0.48 & 4346 & 31172 & 2.2 \\
P3 & 1.46 & 15368 & 83052 & 6.0 \\
A0 & 1.68 & 14712 & 98316 & 7.1 \\
A2 & 3.01 & 52591 & 1147318 & 106.8 * \\
To.ul Area: 1379351 & &
\end{tabular}

Fig. 3: Hemoglobin electrophoresis-capillary electrophoretogram

\section{Conclusion}

With respect to $\mathrm{HbA1C}$, each method has its own advantages and limitations. It is the responsibility of the laboratories to understand, adopt, and optimally utilize these methods based on the needs.

\section{Clinical Significance}

$\mathrm{HbA1c}$ is the gold standard investigation for monitoring patients with diabetes mellitus. NGSP provides traceability and improvement in standardization of methods used for HbA1c measurement. Each method has its own limitations; the most significant from 
the clinical significance point of view is being interfered by the abnormal hemoglobins. Hence each laboratory must understand the strengths and limitations of the available methods in their premises and hold responsibility for reporting clinically relevant results to its patients.

\section{Acknowledgments}

The authors acknowledge the management of MIOT Hospitals for rendering their continuous support in completing the study.

\section{References}

1. http://www.ngsp.org.

2. Rhea JM, Molinaro R. Pathology consultation on HbA1c methods and interferences. Am J Clin Pathol 2014;141(1):5-16. DOI: 10.1309/ AJCPQ23GTTMLAEVL.

3. Simpson WG, Twomey PJ. Reflective testing. J Clin Pathol Reflective testing 2004;57(3):239-240. DOI: 10.1136/jcp.2003.011668.
4. Ryan K, Bain BJ, Worthington D, et al. Significant haemoglobinopathies: guidelines for screening and diagnosis. BJH 2010;149(1):35-49. DOI: 10.1111/j.1365-2141.2009.08054.x.

5. Mosca A, Paleari R, Ivaldi G, et al. The role of haemoglobin A2 testing in the diagnosis of thalassaemias and related haemoglobinopathies. J Clin Pathol 2009;62(1):13-17. DOI: 10.1136/jcp.2008.056945.

6. Joutovsky A, Nesic HJ, Nardi MA. HPLC retention time as a diagnostic tool for hemoglobin variants and hemoglobinopathies: a study of 60000 samples in a clinical diagnostic laboratory. Clin Chem 2004;50(10):1736-1747. DOI: 10.1373/clinchem.2004.034991.

7. Wakeman L, Munro R, Russell C, et al. New reference ranges in haematology for healthy adults using the modern Sysmex XE-2100 automated analyser. Blood 2005;106(11):3740-3745. DOI: 10.1182/ blood.V106.11.3740.3740.

8. Vichinsky E. Hemoglobin E syndromes. Hematol 2007(1):79-83. DOI: 10.1182/asheducation-2007.1.79.

9. HbA1c kit insert. Vitros. Pub No. J55871_EN, version 4:1-18.14.

10. Yedla N, Kuchav MS, Mithal A. Hemoglobin E disease and glycosylated hemoglobin. Indian J Endocrinol Metab 2015;19(5):683-685. DOI: $10.4103 / 2230-8210.163211$. 